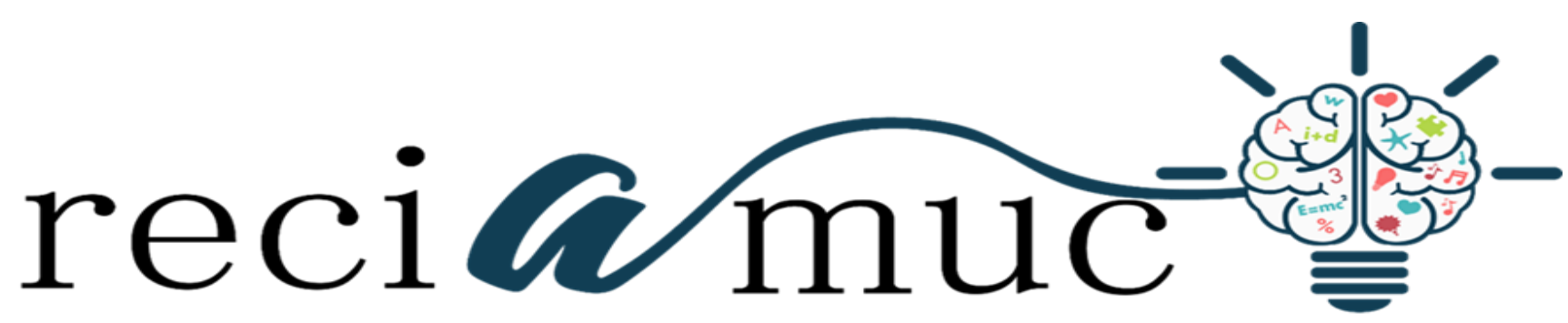

Revista científica de investigación actualización del mundo de las ciencias

\begin{abstract}
Kruzcaya Monserrath Arévalo Sánchez a; Ronald Roberto Reyes Sánchez b; Marjorie Jacqueline Ramírez Ortiz ${ }^{\mathrm{c}}$; Carlos Xavier Villavicencio Bourne ${ }^{\mathrm{d}}$
\end{abstract}

Síndrome de túnel carpiano

Carpal tunnel syndrome

Revista Científica de Investigación actualización del mundo de las Ciencias. Vol. 3 núm., 2, abril, ISSN: 2588-0748, 2018, pp. 827-853

DOI: $10.26820 / \mathrm{reciamuc/3.(2).abril.2019.827-853}$

URL: http://reciamuc.com/index.php/RECIAMUC/article/view/368

Código UNESCO: 3205 Medicina Interna

Tipo de Investigación: Artículo de Revisión

(C) RECIAMUC; Editorial Saberes del Conocimiento, 2019

Recibido: 15/01/2019

Aceptado: 07/02/2019

Publicado: 01/04/2019

Correspondencia: director@reciamuc.com

a. Magister en Gerencia de Salud para el Desarrollo Local; Médico; Investigador Independiente; Guayaquil, Ecuador; kruzcaya.arevalo.md@gmail.com

b. Médico; Investigador Independiente; Guayaquil, Ecuador; triplerrr@ hotmail.es

c. Magister en Seguridad Higiene Industrial y Salud Ocupacional; Medico; Investigador Independiente; Guayaquil, Ecuador; elloreal_22@hotmail.com

d. Médico; Investigador Independiente; Guayaquil, Ecuador; thecharlesone@ hotmail.com 


\section{Síndrome de túnel carpiano}

Vol. 3, núm. 2., (2019)

Kruzcaya Monserrath Arévalo Sánchez; Ronald Roberto Reyes Sánchez; Marjorie Jacqueline Ramírez Ortiz; Carlos Xavier Villavicencio Bourne

\section{RESUMEN}

Los seres humanos para tener niveles de vida óptimos en la sociedad, deben realizar diferentes tareas relacionadas con su profesión u oficio; pero, dicha ejecución afecta su salud; ejemplo de ello, el síndrome de túnel carpiano, visto por las ciencias médicas modernas, como una enfermedad causada por que el nervio mediano que debido a su característica mixta sensitivo - motor formado por la unión de ramas nerviosas de los cordones lateral, y media del plexo braquial; se encuentra atrapado a nivel de la muñeca en su descenso a través del canal o túnel del carpo, siendo un espacio localizado en la base de la palma inmediatamente distal a la muñeca, limitado por los huesos del carpo, con base cóncava y la cuarta por el ligamento transverso del carpo o retináculo flexor. Este síndrome constituye una neuropatía ocupacional relacionada con los factores del trabajo que implican un mayor uso de las manos e involucran movimientos repetitivos de las mismas y la muñeca. A nivel anatomopatológico se observan alteraciones en el tejido conectivo, que incrementa su dolencia como resultado al uso repetitivo de la muñeca durante una actividad laboral, asimismo, muestra afectación en las estructuras anatómicas como: ligamentos, tendones, músculos, lo que influye en el normal funcionamiento del nervio mediano de la mano, su diagnosticado se encuentra identificado como: leve, moderado y grave momento en el cual el médico indica la cirugía. Estas ideas, son las encargadas de orientar el desarrollo del presente artículo con el fin de analizar el síndrome del túnel carpiano, para ello se cumplió con la revisión de diversas literaturas relacionadas con el tema, lo que permitió emplear un método científico esencial que diera camino hacia la construcción de una gama de fuentes relevantes e implementar los criterios de las investigaciones documentales.

Palabras Claves: Síndrome del túnel carpiano; Tejido conectivo; Neuropatía ocupacional; Factores del trabajo. 


\title{
Síndrome de túnel carpiano
}

Vol. 3, núm. 2., (2019)

Kruzcaya Monserrath Arévalo Sánchez; Ronald Roberto Reyes Sánchez; Marjorie Jacqueline Ramírez Ortiz; Carlos Xavier Villavicencio Bourne

\begin{abstract}
Human beings to have optimal levels of life in society, must perform different tasks related to your profession or trade; but, this execution affects their health; example, seen by modern medical science, carpal tunnel syndrome, as a disease that the median nerve that due to its mixed feature sensitive - motor formed by the union of nerve branches from the lateral cord, and a half of the brachial plexus; is trapped at the wrist in his descent through the channel or carpal tunnel, being a space located at the base of the Palm immediately distal to the wrist, constrained by the bones of the Carpus, with concave base and the fourth by the transverse ligament of the Carpus or flexor retinaculum. This syndrome is an occupational neuropathy related factors of work that involve greater use of hands and involve repetitive motions of the same and the wrist. Level pathological there are alterations in the connective tissue, which increases his ailment as a result to the repetitive use of the wrist during a work activity, also shows involvement in the anatomical structures such as: ligaments, tendons, muscles, this affects the normal functioning of the median nerve in the hand, his diagnosed is identified as: mild, moderate and severe moment in which the doctor suggests surgery. These ideas are responsible for guiding the development of this article in order to analyze the carpal tunnel syndrome, this was met with various topic-related literature review, allowing you to use a scientific method essential to give road towards the construction of a range of relevant sources and implementing the criteria of the documentary research.
\end{abstract}

Key Words: Síndrome del túnel carpiano; Tejido conectivo; Neuropatía ocupacional; Factores del trabajo. 


\section{Síndrome de túnel carpiano}

Vol. 3, núm. 2., (2019)

Kruzcaya Monserrath Arévalo Sánchez; Ronald Roberto Reyes Sánchez; Marjorie Jacqueline Ramírez Ortiz; Carlos Xavier Villavicencio Bourne

\section{Introducción.}

En la actualidad las enfermedades osteomuscular ocupan en el primer lugar en las enfermedades profesionales, siendo el síndrome de túnel carpiano la primera causa de morbilidad profesional, con causas que aumenta cada año, éste síndrome ha sido definido como un conjunto de signos y síntomas que se producen por la compresión del nervio mediano en el canal del carpio, encargado de trasmitir señales que controlan los movimientos de los dedos en las manos, su inflamación puede ser causada por traumatismo o esfuerzos manuales repetitivos que generan hormigueo, sensación adormecimiento, dolores nocturnos que dificultan el sueño y pérdida de destreza manual.

De este modo, se entiende que el Síndrome de Túnel del Carpo (STC), representa una de las patologías que afectan a las personas en su capacidad laboral, la misma se encuentra caracterizada por diferentes manifestaciones relacionadas directamente con el nervio mediano que sufre una irritación frecuentemente por atrapamiento a la altura del túnel carpiano, por ello, los trabajadores que se desempeñan en labores vinculadas con el uso de computadoras, aseo, operadores de la industria, pianista, barrenderos, empacadores, secretarias, actividades del hogar entre otras, donde se amerite el uso repetitivo de movimientos donde se involucra las manos; los mismos manifiestan fatiga, bajo desempeño, dolor, molestias generales.

Según las consideraciones anteriores, se evidencia que la presencia de esta enfermedad, se encuentra estrechamente relacionada con las actividades profesionales u oficios que las personas llevan a cabo, donde exigen de manera repetitiva el uso de las manos, es decir, representa una de las neuropatías periféricas comprensivas más comunes, visualizándose como una patología 


\section{Síndrome de túnel carpiano}

Vol. 3, núm. 2., (2019)

Kruzcaya Monserrath Arévalo Sánchez; Ronald Roberto Reyes Sánchez; Marjorie Jacqueline Ramírez Ortiz; Carlos Xavier Villavicencio Bourne

laboral. En relación a este planteamiento, Quiroz y Hernández (2017) demostraron que "el 86\% de la población que realiza movimientos repetitivos de mano y muñeca en un $91 \%$ van a tener dolor, hormigueo y molestias que contribuyen a la pérdida de la fuerza en la mano". (p.11). Estos hallazgos, llevan a entender, que la prevalencia del síndrome de túnel carpiano, es elevado, además su sintomatología agrega en el desempeño laboral del individuo consecuencias negativas como resultado a las diferentes dolencias que experimenta e imposibilidad su capacidad para cumplir con eficiencia el trabajo correspondiente.

En razón de lo anterior, el síndrome del túnel carpiano (STC) o conocido también como parálisis tardía del nervio mediano es una lesión compresiva de dicho nervio por múltiples causas. Asimismo, es definida como una neuropatía periférica que presenta manifestaciones sensitivas, motoras y tróficas y por consiguiente afectará desde el punto de vista físico, psíquico, social y laboral a la persona. De acuerdo con Silva (2018) destaca que "esta enfermedad es frecuente en mujeres de 45 años, tendencia en la mano dominante, su prevalencia es de $4 \%$ y $8 \%$ en individuos de 55 años, factores de riesgos personas mayores de 40 años, obesos, femeninos y diabéticos". (p.6). Es decir, el surgimiento de este síndrome va a estar determinado por la presencia de factores relacionados con el sexo, edad, afectando con mayor tendencia a la mano dominante; además de sumarse sus condiciones físicas relativas a la obesidad y diabetes.

Cabe destacar que, esta neuropatía compresiva del nervio mediano a nivel del túnel del carpo ocurre de forma secundaria, debido a una posición inadecuada de la muñeca y mano, en la presión local en la cara anterior de la mano por movimientos forzados y repetitivos o vibración. Es más frecuente en los profesionales en la mano dominante, puede estar relacionada con otras 


\section{Síndrome de túnel carpiano}

Vol. 3, núm. 2., (2019)

Kruzcaya Monserrath Arévalo Sánchez; Ronald Roberto Reyes Sánchez; Marjorie Jacqueline Ramírez Ortiz; Carlos Xavier Villavicencio Bourne

afecciones como: Lesiones ocupantes del espacio, enfermedades del tejido conectivo, reumatológicas por depósitos de microcristales, infecciones, enfermedades metabólicas, endocrinas, lesiones iatrogénicas y misceláneas (embarazo, amiloidosis, diálisis, fracturas) y enfermedades ocupacionales como: carniceros, músicos, mecanógrafos y deportistas entre otros, existe una relación entre los factores ergonómicos como: repetición, intensidad de la fuerza y posturas extreman de la articulación.

De igual forma, se puede complementar que este síndrome al presentarse durante el embarazo, se resuelve después del parto y debe tratarse conservadoramente; aunque existe un tratamiento a seleccionar con la inyección de inyección de dexametasona que puede ser aplicada en el 3er trimestre. También es común en mujeres con menopausia y con cáncer de mama, recibiendo terapia hormonal y en muchos casos han necesitado la liberación quirúrgica. Entre los síntomas más comunes se encuentran las parestesias y el dolor. Este dolor neuropático es causado por diferentes eventos como es la compresión, infiltración, isquemia o daño metabólico de la neurona; es decir, la presencia de este dolor neuropático, se origina como resultado al comprimido del nervio, el cual se inflama por un proceso isquémico e inmunológico que provoca el daño a las fibras nerviosas y clínicamente se manifiesta por un dolor neuropático. Asimismo, el estrés oxidativo tiene que ver en la patogénesis del dolor neuropático, de hecho, la alteración del mecanismo de reducción del oxígeno con excesiva producción de radicales libres produce una isquemia con disminución de la suplencia de nutrientes para las células nerviosas.

De igual manera, Silva (ob.cit), destaca que en Ecuador En Ecuador según el Instituto Nacional de Estadísticas y Censos “el 32\% de la población padecen síndrome del túnel carpiano y 


\section{Síndrome de túnel carpiano}

Vol. 3, núm. 2., (2019)

Kruzcaya Monserrath Arévalo Sánchez; Ronald Roberto Reyes Sánchez; Marjorie Jacqueline Ramírez Ortiz; Carlos Xavier Villavicencio Bourne

no sólo quienes trabajan con computadoras más de 10 horas al día son propensos a padecer esta patología."(p.8). Es decir, hay otros empleos que, por demandar un movimiento repetitivo de la mano y muñeca, pueden derivar en este padecimiento masajistas, carpinteros, cajeros de supermercados, encargados de línea de montaje, empacadores, violinistas, pianistas, tenistas y empleadas de maquiladoras, muestran una alta proclividad a este tipo de afecciones.

Según los planteamientos anteriores, se puede acotar que la presencia de este síndrome de túnel carpiano, se hace evidente en aquellas personas o profesionales que realizan actividades laborales que exigen una mayor utilización de las manos, en consecuencia, se inflama el nervio y se inicia un proceso de síntomas relativas a dolor neuropático, en algunos casos produce pérdida de la sensibilidad, hinchazón, hormigueo, poca fuerza para llevar a cabo otras actividades complementarias de la vida diaria, su tendencia es más frecuente en mujeres, personas obesas o que presente diabetes; clínicamente su diagnóstico se realiza debido a la presencia del dolor agudo que indica el paciente, existe tratamiento y utilización de la cirugía como abordaje final. Es decir, hoy se reconoce como una patología contraída a consecuencia del trabajo ejecutado, lo cual implica que queda cubierta en los mismos términos que un accidente de trabajo por la mutua de trabajo o por la seguridad social.

Por lo tanto, lo expuesto, sirve de apoyo referencial para el desarrollo del presente artículo, mediante el cual se busca analizar el síndrome de túnel carpiano, por ello, mediante los respectivos argumentos, se busca adecuar los espacios temáticos en verdaderos puntos de vista encargados de dar la oportunidad de valorar con nuevos criterios los referidos tópicos seleccionados, para lo cual, es fundamental ubicarlo en un área científica. 


\section{Síndrome de túnel carpiano}

Vol. 3, núm. 2., (2019)

Kruzcaya Monserrath Arévalo Sánchez; Ronald Roberto Reyes Sánchez; Marjorie Jacqueline Ramírez Ortiz; Carlos Xavier Villavicencio Bourne

\section{Método.}

Los procesos relacionados con la cientificidad se encuentran determinados por ser procedimientos reflexivos- críticos que funcionan a partir de un sistema informativo para proponer nuevos hechos, datos, relaciones e ideas en cualquier ámbito del conocimiento científico. Por lo tanto, la recopilación de la información resulta de carácter relevante y fidedigna; pero que al mismo tiempo no puede considerarse absolutamente verdadera, pues, en este particular se encuentra vinculada con un análisis acerca del síndrome de túnel carpiano, donde existe la necesidad permanente de nuevas investigaciones encargadas de resaltar mediante la experimentación los hallazgos expuestos anteriormente; en consecuencia para resolver este esquema, se hace inminente la presencia de un método.

En tal sentido, Callejo (2019) define al método científico como "una palanca crítica que estima un modelo previo para lograr medir, analizar, describir, interpretar, resolver y emplear sus direcciones desde la perspectiva lógica y fijar una posición crítica ante los hechos”.(p. 56). Es decir, la escogencia de un método, hace posible que el investigador postule sus propios criterios dentro de una concepción lógica, para lo cual, es determinante seleccionar los planteamientos de otros teóricos e interpretar correlativamente para llegar a indicar las conclusiones del tema.

De este modo, se estima como método que dirige el accionar científico del presente artículo, el tipo explicativo, mediante el cual se asegura no sólo la lectura e interpretación de los elementos que caracterizan al síndrome de túnel carpiano, sino hace posible traspolar nuevas ideas desde una visión global. Tal como lo plantea Mora (2019) “un método explicativo asegura la transformar de dogmas o principios en principios que subyacentes en el proceso investigativo". (p. 


\section{Síndrome de túnel carpiano}

Vol. 3, núm. 2., (2019)

Kruzcaya Monserrath Arévalo Sánchez; Ronald Roberto Reyes Sánchez; Marjorie Jacqueline Ramírez Ortiz; Carlos Xavier Villavicencio Bourne

69). De allí, que su selección y aplicación en este proceso investigativo, hizo posible, verificar los contenidos y darle la respectiva organización para convertirlos en nuevos esquemas que aporten informaciones reales al campo en estudio.

Tipo de Investigación

La combinación de este método con el tipo de investigación facilita el cumplimiento de las actividades reflexivas encargadas de asignarle el respectivo bagaje informativo desde una perspectiva global. Por lo tanto, para configurar su estructura crítica se cumple con la ubicación en el campo de las investigaciones documentales. Definida por Callejo (ob.cit) como "un argumento general, que busca plasmar ideas, reorientar un proceso previo con otros aportes para convertirlo en un proceso realmente monográfico". (p.61). Estas ideas, permiten entender que al seleccionar la investigación documental, el investigador, tiene posibilidades de recopilar informaciones expuestas por otros autores, convertirlas en nuevas apreciaciones y generar de esta combinación la construcción de un cuerpo teórico relevante para la ciencia.

\section{Fuentes Documentales}

Los procesos investigativos documentales, deben mantener su condición innovadora, para ello necesitan incorporar diferentes fuentes referenciales que le ayuden a recabar los aportes de mayor significación en la interpretación de los argumentos previamente expuestos, es decir, lograr nuevos conocimientos, en consecuencia, el investigador puede obtener de trabajos anteriores y comprobarlos para así replantear sus apreciaciones. En consecuencia, es importante agregar lo citado por Mora (ob.cit) las fuentes documentales "son materiales presentes en diferentes 


\section{Síndrome de túnel carpiano}

Vol. 3, núm. 2., (2019)

Kruzcaya Monserrath Arévalo Sánchez; Ronald Roberto Reyes Sánchez; Marjorie Jacqueline Ramírez Ortiz; Carlos Xavier Villavicencio Bourne

escenarios que al ser combinados ayudan al investigador a plantear con objetividad los cambios que considera válidos para el hecho científico”. (p. 71).

Al analizar la conceptualización citada, se puede entender que para el desarrollo de los eventos teóricos que estructuran al artículo en desarrollo, deben ser planificados en función a las lecturas previas de aquellos autores anteriores que consideraron la temática como un punto de interés, en otras palabras, se revisaron sus consideraciones, para replantear nuevos criterios agregando el método explicativo y así construir cada momento en función a los aspectos que se encuentran relacionados con el tópico de forma crítica- reflexiva, apoyado en el uso de los recursos tecnológicos como nuevos elementos y dar la respectiva orientación al contenido que fue debidamente valorado.

\section{Técnica de Recolección de Información}

El trabajo científico para generar confianza, debe responder a la presencia de una objetividad básica, mediante este proceso se puede asegurar con anticipación el reporte bibliográfico obtenido, en otras palabras, hay que considerar técnicas como: lectura, revisión interpretativa, argumentación de tópicos, análisis reflexivo, todos ellos en correspondencia con el método explicativo, para así alcanzar el respectivo nivel descriptivo del proceso investigativo. Estas consideraciones, le dan respuesta a lo citado por Mora (ob.cit) en una investigación documental "el investigador debe emplear diferentes técnicas de recopilación de información, combinarlas para responder a las interrogantes planteadas”. (p. 71). 


\section{Síndrome de túnel carpiano}

Vol. 3, núm. 2., (2019)

Kruzcaya Monserrath Arévalo Sánchez; Ronald Roberto Reyes Sánchez; Marjorie Jacqueline Ramírez Ortiz; Carlos Xavier Villavicencio Bourne

De lo antes expuesto, se puede decir que mediante la utilización de las diferentes técnicas centradas en la lectura crítica- reflexiva, se logra indicar mediante valoraciones explícitas los nuevos argumentos que surgen como resultado de interpretar en forma precisa y coherente a los tópicos tratados, de esta forma, construye sus contenidos desde su propia visión, para así darle la respectiva consistencia objetiva e innovadora que amerita el hecho investigativo.

\section{Resultados.}

El análisis que caracteriza al estudio en curso lleva a indicar a continuación los diferentes aportes encargados de describir el tema previamente seleccionado, por lo tanto, su organización se hizo en un grupo de eventos, debido a las condiciones temática que lo caracterizan.

\section{Anatomía de la Mano}

El esqueleto de la mano se compone de 27 huesos, divididos en tres grupos: el carpo, metacarpianos y falanges. En su conjunto, adoptan la forma de arcos, dos transversales (a nivel del carpo y metacarpianos) y los arcos longitudinales digitales; la mano, es una articulación condílea, pues permite realizar movimientos de flexión, extensión, desviación radial y cubital y circunducción. La misma puede, considerarse un área fija, integrada por la hilera distal del carpo (trapecio, trapezoide, hueso grande y ganchoso) y los metacarpianos segundo y tercero, un área móvil, integrada por la hilera proximal del carpo (escafoides, semilunar y piramidal), los metacarpianos primero, cuarto y quinto, así como las falanges. El pisiforme, clásicamente englobado en la hilera proximal, está en realidad situado en un nivel más palmar, y actúa a manera de hueso sesamoideo, insertándose en él el tendón cubital anterior. 


\section{Síndrome de túnel carpiano}

Vol. 3, núm. 2., (2019)

Kruzcaya Monserrath Arévalo Sánchez; Ronald Roberto Reyes Sánchez; Marjorie Jacqueline Ramírez Ortiz; Carlos Xavier Villavicencio Bourne

Cabe destacar que, una característica esencial de la mano humana es la capacidad para oponer el dedo pulgar a los restantes dedos, gracias a la especial configuración de la articulación del trapecio. Además, tiene numerosos músculos, ligamentos, y vainas. Los músculos son estructuras que se contraen y permiten el movimiento de los huesos de la mano. Los ligamentos son tejidos fibrosos que ayudan a unir las articulaciones de la mano. Las vainas son estructuras tubulares que rodean parte de los dedos.

De los veintisiete huesos, que posee cada mano, ocho son en el metacarpo, cinco metacarpianos y un total de catorce falanges. Aunque se le considera como una sola articulación, la muñeca es en realidad una articulación compuesta, con movimiento global, debido a las interacciones entre los huesos individuales del carpo, así como interacciones distales con las bases de los metacarpianos y en dirección proximal con la superficie articular distal de cubito y radio. Los ocho huesos del carpo se organizan en dos hileras una proximal y una distal. De radial a cubital la hilera proximal está compuesta de los huesos escafoides, semilunar, piramidal y pisiforme (aunque el pisiforme es un hueso sesamoideo ubicado en el interior del tendón flexor carpiulnaris. La hilera distal la forman, el trapecio, trapezoide, hueso grande y ganchoso; de tal manera que el escafoides funciona como punto de unión entre las dos hileras.

La articulación radiocarpiana está formada por la articulación de la hilera proximal del carpo con la superficie articular distal del radio y el complejo fibrocartilaginoso triangular. La superficie articular distal del radio es cóncava y está inclinada en dos planos, con un promedio de $11^{\circ}$ de inclinación palmar en el plano sagital y $22^{\circ}$ de inclinación cubital en el plano coronal. Una prominencia interfacetaria denominada cresta fibrocartilaginosa sagital, identifica la separación 


\section{Síndrome de túnel carpiano}

Vol. 3, núm. 2., (2019)

Kruzcaya Monserrath Arévalo Sánchez; Ronald Roberto Reyes Sánchez; Marjorie Jacqueline Ramírez Ortiz; Carlos Xavier Villavicencio Bourne

entre las fosas semilunar y escafoides sobre el extremo distal del radio. La superficie articular proximal del piramidal es relativamente plana, pero en gran parte no se articula con otros huesos.

En cuanto a las articulaciones carpometacarpianas de los dedos, excluyendo en pulgar, están recubiertas de una gruesa cápsula y cobertura ligamentaria. El segundo y tercer metacarpianos se encuentran rígidamente fijos al trapezoide y hueso grande, proporcionando una base estable sobre la cual el pulgar y los dos metacarpianos cubitales giran durante el movimiento de la mano. La segunda articulación tiene sólo $1^{\circ} \mathrm{o} 2^{\circ}$ de movimiento, y la tercera no permite más de $3^{\circ}$. En cambio, la cuarta y quinta articulaciones carpometacarpianas permiten de 10 a $15^{\circ}$ de movimiento respectivamente.

Es decir, la primera articulación carpometacarpiana, se distingue del resto de los dedos por su capacidad de moverse en grado importante en cuatro planos. La articulación trapeciometacarpiana tiene una configuración bicóncava (en silla de montar) y depende en buena medida de la restricción ligamentaria para proporcionarle estabilidad a pesar de su arco de movimientos. Mientras que las falanges proximales de los dedos 2 a 5 forman una articulación de tipo condileo con sus metacarpianos, mientras que la primera articulación metacarpofalángica es más una diartrosis, con poco movimiento en el plano radiocubital coronal y con alta variabilidad en flexión y extensión. Las articulaciones interfalángicas funcionan como diartrosis, que permiten una considerable flexión, mientras que limitan junto con su sistema ligamentario, en forma considerable, la extensión a este nivel.

Es importante acotar que las uniones de los diversos huesos de la mano, están reforzadas por un grupo de sistema ligamentario que le permite su función mientras ayuda a mantener sus 


\section{Síndrome de túnel carpiano}

Vol. 3, núm. 2., (2019)

Kruzcaya Monserrath Arévalo Sánchez; Ronald Roberto Reyes Sánchez; Marjorie Jacqueline Ramírez Ortiz; Carlos Xavier Villavicencio Bourne

relaciones anatómicas. Por lo tanto, los ligamentos de la muñeca se dividen en intrínsecos y extrínsecos. Estos últimos se insertan en los huesos del carpo o proximales o distales a ellos, mientras que los intrínsecos se insertan por completo en los límites del carpo. Mientras que los extrínsecos se clasifican como radiocarpianos y cubitocarpianos. Desde la apófisis estiloides del radio, de ulnar a radial, se describen los ligamentos el radioescafoideo-hueso grande, el radioulnar largo, el radio escafosemilunar y el radioulnar corto.

El ligamento radioulnar largo se ha llamado ligamento radioulnar piramidal, no obstante, nuevos datos sugieren que su breve trayecto sobre la cara palmar del semilunar hacia el piramidal no es suficiente para justificar ese nombre. De igual forma, aunque históricamente el ligamento radioescafosemilunar se ha considerado como el principal restrictor de la flexión del escafoides o subluxación en rotación, estudios recientes1 muestran que tal ligamento no es tejido ligamentario conectivo verdadero, sino un vínculo neurovascular, que tiene mínima contribución mecánica. El ligamento radioulnar corto nace en el borde palmar de la fosa semilunar y se dirige distalmente para insertarse en la cara proximal de la apófisis unciforme del semilunar. En dirección radial, este ligamento se separa del ligamento radioulnar largo, por la penetración de radio escafosemilunar, a través de la cápsula radiocarpiana palmar. En dirección cubital, se combinan con las fibras que se originan en el reborde palmar de complejo fibrocartilaginoso triangular y se inserta en el semilunar. Este ligamento parece ser el principal estabilizador del semilunar. Los ligamentos cubitoulnar y cubitopiramidal se originan en el complejo fibrocartilaginoso triangular y se insertan en la cara palmar de semilunar y piramidal respectivamente. 


\section{Síndrome de túnel carpiano}

Vol. 3, núm. 2., (2019)

Kruzcaya Monserrath Arévalo Sánchez; Ronald Roberto Reyes Sánchez; Marjorie Jacqueline Ramírez Ortiz; Carlos Xavier Villavicencio Bourne

En cuanto al soporte de las articulaciones metacarpofalángicas, a cada lado de las mismas está dado por la lámina palmar común y el complejo ligamentario lateral. Dado que las cabezas de los metacarpianos se ensanchan en dirección de dorsal a palmar, los ligamentos se mantienen fijos en flexión. Tal efecto obliga a la inmovilización de las articulaciones metacarpofalángicas en flexión, a fin de evitar el acortamiento del ligamento lateral, relativamente laxo, lo cual se presenta cuando la articulación metacarpofalángica está en extensión. Entre los ligamentos intrínsecos figuran los interóseos, así como el ligamento en $\mathrm{V}$ o deltoideo (escafopiramidal o intercarpiano dorsal); este se origina en la superficie dorsal del cuello y el polo distal del escafoides, y cruza en dirección transversa sobre el semilunar para insertarse en el piramidal, para cumplir una importante fusión en la estabilización transversa de la hilera proximal. En la superficie palmar del carpo, comenzando en la porción radial, se cuentan entre los ligamentos intrínsecos el escafotrapecialtrapezoidal, escafoides-hueso grande, piramidoganchoso, piramidal-hueso grande, escafosemilunar y el lunopiramidal.

Cabe agregar, que la movilidad de la mano está dada por músculos y tendones flexores y extensores. Cuando el origen de éstos está en el antebrazo se habla de músculos extrínsecos, por el contrario, cuando se originan en la misma mano se habla del sistema muscular intrínseco. León (2015) destaca que los músculos flexores extrínsecos, se originan aproximadamente en el antebrazo, volviéndose tendinosos en los tercios medio y distal del mismo. En el túnel del carpo el nervio mediano se acompaña de 9 tendones, 4 superficiales, 4 profundos y el flexor pollicis longus. Los flexor carpi radialis y carpi ulnaris se insertan en la base del segundo hueso metacarpiano, hueso ganchoso y quinto metacarpiano respectivamente. El tendón del flexor carpi 


\section{Síndrome de túnel carpiano}

Vol. 3, núm. 2., (2019)

Kruzcaya Monserrath Arévalo Sánchez; Ronald Roberto Reyes Sánchez; Marjorie Jacqueline Ramírez Ortiz; Carlos Xavier Villavicencio Bourne

ulnaris, envuelve al pisiforme, lo cual ofrece ventajas biomecánicas a su función como flexor de la muñeca y desviador cubital.

Figura $\mathbf{N}^{\circ} 1$ Músculos de la Mano

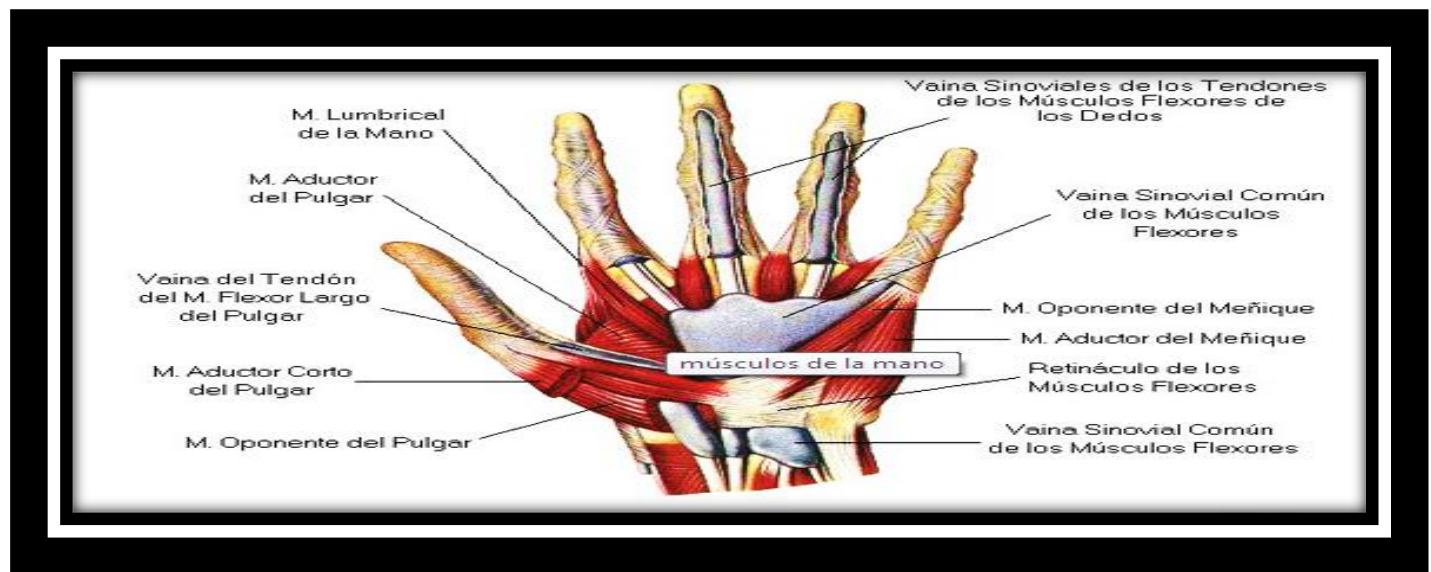

Fuente: León (ob.cit)

Además, puesto que la flexión de la muñeca y la desviación cubital son sinérgicas, este tendón es el más importante de los dos flexores de la muñeca; por lo que la transferencia tendinosa del flexor carpi radialis como donante conlleva poco o ningún daño funcional por el uso del tendón. El ligamento transverso del carpo, se extiende desde el escafoides y el trapecio en la parte radial, hasta el hueso ganchoso en la porción medial, formando el techo del túnel del carpo. En el interior de éste túnel los tendones flexores de los dedos, yacen profundos respecto al nervio mediano, pero la inflamación del tendón, puede contribuir y en algunos casos causar, síndrome del túnel del carpo. Después de pasar por el surco, el tendón flexor atraviesa la palma y entra en la zona de la vaina osteofibrosa, antes de insertarse en la falange media en el caso del tendón superficial, y sobre la falange distal en el caso del tendón profundo. 


\section{Síndrome de túnel carpiano}

Vol. 3, núm. 2., (2019)

Kruzcaya Monserrath Arévalo Sánchez; Ronald Roberto Reyes Sánchez; Marjorie Jacqueline Ramírez Ortiz; Carlos Xavier Villavicencio Bourne

\section{Síndrome de Túnel Carpiano}

El hombre para su desarrollo ha utilizado la mano como principal herramienta, lo cual constituyó un avance decisivo en su transformación, integrándose definitivamente como un ser social. Mediante la mano realiza una variedad de funciones en las cuales corre grandes riesgos de padecer una afección, debido al sobre uso de la misma. Dentro de ellas, se destaca el síndrome de túnel carpiano, que es una neuropatía periférica que ocurre cuando el nervio mediano, se presiona o se atrapa dentro del túnel carpiano, a nivel de la muñeca, afecta hasta a un 3\% de la población general; además, representa un lugar destacado en cuanto a salud ocupacional se refiere, en Estados Unidos la incidencia actual es de $0.1 \%$ y en la población trabajadora del $15 \%$ al $20 \%$. Esto lleva a resaltar que, las enfermedades ocupacionales son patologías contraídas como resultados de la exposición a factores de riesgo como agentes físicos, químicos, biológicos y ergonómicos factores inherentes a la actividad laboral dentro de estas están las enfermedades por esfuerzos repetitivos que son las más comunes.

Es importante resaltar que, el túnel carpiano es un paso estrecho de ligamentos y huesos en la base de la mano que contiene nervios y tendones, que pueden engrosarse e irritar a otras estructuras hinchadas que estrechan el túnel para causar compresión de los nervios. Los síntomas suelen comenzar gradualmente. A medida que empeoran, sostener objetos puede tornarse difícil. Por ello, su detección y tratamiento temprano son importantes para prevenir daño permanente a los nervios. La manera de diagnosticarlo es a través de un examen físico y exámenes específicos de los nervios. El tratamiento incluye reposo de las manos, uso de férulas, medicinas para el dolor y la inflamación y, a veces, cirugía. 


\section{Síndrome de túnel carpiano}

Vol. 3, núm. 2., (2019)

Kruzcaya Monserrath Arévalo Sánchez; Ronald Roberto Reyes Sánchez; Marjorie Jacqueline Ramírez Ortiz; Carlos Xavier Villavicencio Bourne

Al respecto, León (ob.cit), destaca que el nervio mediano proporciona sensación, movimiento al lado de la mano en el que se encuentra el dedo pulgar, incluye la palma de la mano, dedo pulgar, índice, medio, anular y pulgar. (p. 26) Por lo tanto, la zona en la muñeca donde el nervio entra en la mano se llama túnel carpiano. Este normalmente es angosto, cualquiera inflamación puede pellizcar al nervio y causar dolor, entumecimiento, hormigueo o debilidad, identificado como síndrome de túnel carpiano; también puede ser causado por hacer el mismo movimiento de la mano y la muñeca una y otra vez. El uso de herramientas manuales que vibran también puede llevar a este síndrome.

En consecuencia, la sintomatología que cuenta el paciente es bastante característica y ayuda al médico a orientar el diagnóstico. Tras la sospecha clínica, se deberá realizar una exploración de la sensibilidad y fuerza de la mano, así como maniobras que desencadenen la sintomatología. Para confirmar el diagnóstico y valorar el grado de afectación del nervio mediano (leve, moderado o severo) habitualmente se solicita un estudio neurofisiológico consistente en un electromiograma (EMG) y un estudio de conducción nerviosa (ECN). El síndrome del túnel carpiano puede asociarse a enfermedades endocrinológicas, reumáticas, tratamiento con estrógenos, embarazo, algunos tumores, entre otros.; es por eso que, si existe la sospecha de estas enfermedades, el diagnóstico puede complementarse con pruebas analíticas o de imagen que ayuden a encontrar la causa.

De igual manera, Garayova (2016) plantea que el túnel carpiano "es un canal o corredor en la cara anterior o flexora de la muñeca, situado entre los huesos de la muñeca y el ligamento anular del carpo, por el cual pasan los tendones flexores de los dedos y el nervio mediano.” (p.36). De 


\section{Síndrome de túnel carpiano}

Vol. 3, núm. 2., (2019)

Kruzcaya Monserrath Arévalo Sánchez; Ronald Roberto Reyes Sánchez; Marjorie Jacqueline Ramírez Ortiz; Carlos Xavier Villavicencio Bourne

allí, que la aparición del síndrome, va a estar caracterizado por la presencia de dolor, pues, se produce daño del nervio mediano. Al existir cualquier causa, un aumento de la presión dentro de ese túnel carpiano se puede producir una lesión en dicho nervio. Los casos leves pueden evolucionar favorablemente con tratamiento mediante inmovilización de la muñeca o con la inyección de corticoides. En casos más avanzados, la solución siempre es quirúrgica, liberando el nervio atrapado en el túnel del carpo.

De lo antes expuesto, se desprende que el síndrome del túnel carpiano es un trastorno que causa entumecimiento, hormigueo y otros síntomas en la mano y el brazo. Este síndrome se manifiesta por la compresión del nervio del túnel carpiano, conducto estrecho ubicado en la muñeca, del lado de la palma de la mano. La anatomía de la muñeca, algunos problemas de salud y posiblemente los movimientos repetitivos de la mano pueden contribuir a la aparición del síndrome del túnel carpiano.

Figura $\mathbf{N}^{\circ} 2$ Túnel Carpiano

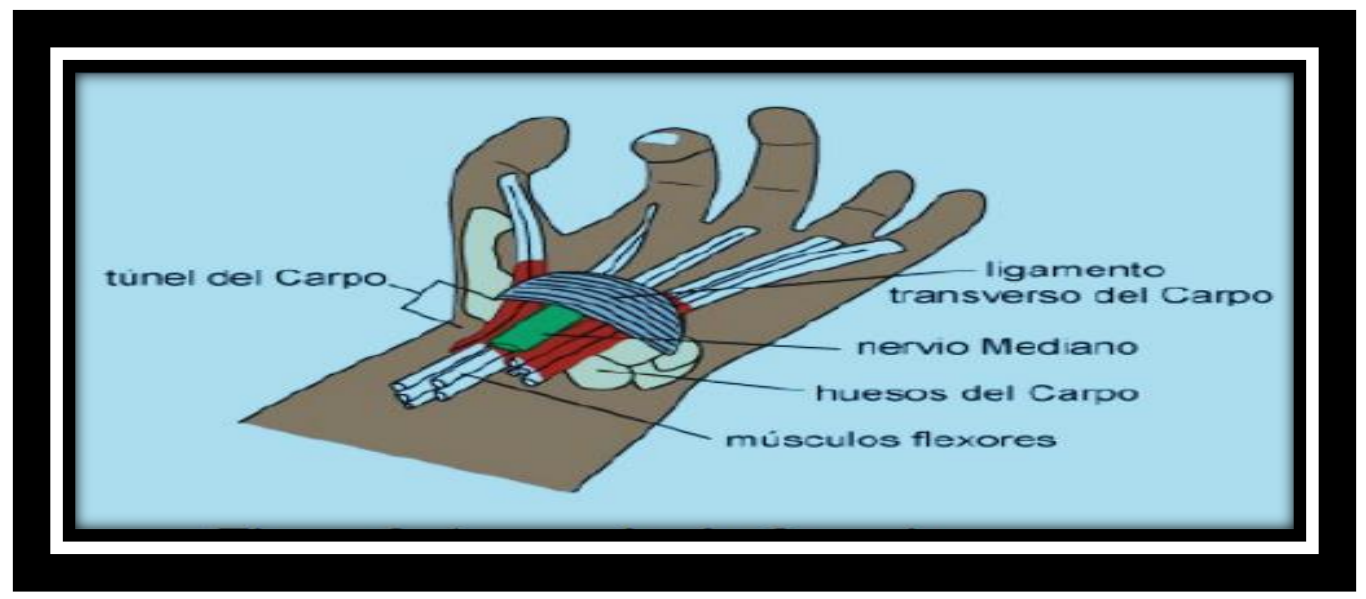

Fuente: León (ob.cit) 


\section{Síndrome de túnel carpiano}

Vol. 3, núm. 2., (2019)

Kruzcaya Monserrath Arévalo Sánchez; Ronald Roberto Reyes Sánchez; Marjorie Jacqueline Ramírez Ortiz; Carlos Xavier Villavicencio Bourne

Por lo general, los síntomas del síndrome del túnel carpiano comienzan gradualmente. Entre ellos, se encuentran entumecimiento u hormigueo en los dedos pulgar, índice y medio que aparece y desaparece. El síndrome del túnel carpiano también puede provocar molestia en la muñeca y la palma de la mano. Los síntomas frecuentes del síndrome del túnel carpiano según León (ob.cit) son:

Hormigueo o entumecimiento: Puedes sentir hormigueo y entumecimiento en los dedos o en las manos. Por lo general, los dedos afectados son el pulgar, el índice y el medio o anular, pero no el meñique. En ocasiones, se siente como si hubieras recibido un choque eléctrico en esos dedos. La sensación se puede extender desde la muñeca hasta el brazo. Los síntomas suelen aparecer cuando sostienes el volante, el teléfono o el periódico. La sensación puede hacer que te despiertes. Muchas personas sacuden las manos para intentar aliviar los síntomas. La sensación de entumecimiento puede volverse constante con el tiempo.

Debilidad. Puedes notar debilidad en la mano y una tendencia a dejar caer objetos. Esto puede ser por el entumecimiento de la mano o la debilidad de los músculos del dedo pulgar que hacen el movimiento de pinza, los cuales también controlan el nervio mediano.

Este mismo autor, hace unas acotaciones en cuanto a los factores que originan el respectivo síndrome de túnel carpiano, los mismos pueden aumentar las posibilidades de padecer o agravar la lesión al nervio mediano. Algunos de ellos son:

Factores anatómicos: Una fractura o dislocación de muñeca, o artritis que deforma los huesos pequeños de la muñeca, puede alterar el espacio dentro del túnel carpiano y ejercer presión 


\section{Síndrome de túnel carpiano}

Vol. 3, núm. 2., (2019)

Kruzcaya Monserrath Arévalo Sánchez; Ronald Roberto Reyes Sánchez; Marjorie Jacqueline Ramírez Ortiz; Carlos Xavier Villavicencio Bourne

sobre el nervio mediano. Las personas que tienen túneles carpianos más pequeños tienen más probabilidades de padecer este síndrome.

Sexo: El síndrome del túnel carpiano suele ser más frecuente en las mujeres. Esto podría deberse a que la zona del túnel carpiano es relativamente más pequeña en las mujeres que en los hombres. Las mujeres que tienen el síndrome del túnel carpiano también pueden tener túneles carpianos más pequeños que las que no padecen este trastorno.

Afecciones que provocan lesiones a los nervios: Algunas enfermedades crónicas, como la diabetes, aumentan el riesgo de lesión a los nervios, como la lesión al nervio mediano.

Enfermedades inflamatorias: Las enfermedades caracterizadas por la inflamación, como la artritis reumatoide, pueden afectar el revestimiento que rodea los tendones de la muñeca y ejercer presión sobre el nervio mediano.

Obesidad: La obesidad constituye un factor de riesgo importante de padecer síndrome del túnel carpiano.

Alteraciones en el equilibrio de los líquidos corporales: La retención de líquidos puede aumentar la presión dentro del túnel carpiano, lo que irrita el nervio mediano. Esto es frecuente durante el embarazo y la menopausia. El síndrome del túnel carpiano asociado al embarazo generalmente se resuelve solo después del embarazo. 


\section{Síndrome de túnel carpiano}

Vol. 3, núm. 2., (2019)

Kruzcaya Monserrath Arévalo Sánchez; Ronald Roberto Reyes Sánchez; Marjorie Jacqueline Ramírez Ortiz; Carlos Xavier Villavicencio Bourne

Otras afecciones: Algunas afecciones, como la menopausia, la obesidad, los trastornos de la tiroides y la insuficiencia renal, pueden aumentar las posibilidades de padecer el síndrome del túnel carpiano.

Factores del lugar de trabajo: Es posible que el trabajo con herramientas que vibran o en una cadena de montaje que requiere movimientos de flexión de la muñeca prolongados y repetitivos genere una presión dañina sobre el nervio mediano o empeore la lesión existente al nervio.

No obstante, la evidencia científica es contradictoria y estos factores no se han establecido como causas directas del síndrome del túnel carpiano. Varios estudios han evaluado la posible relación entre el uso de la computadora y el síndrome del túnel carpiano. Sin embargo, no se ha hallado suficiente evidencia de calidad y consistente para respaldar el uso extendido de la computadora como un factor de riesgo del síndrome del túnel carpiano, aunque este puede causar una forma diferente de dolor en la mano.

Ante la presencia de todos estos factores, al ser diagnosticado con la presencia del síndrome de túnel carpiano, es importante que el paciente cumpla una serie de actividades preventivas con el fin de lograr una mejoría. Aunque no hay estrategias demostradas para prevenir el síndrome del túnel carpiano, pero puedes aliviar la tensión en las manos y en las muñecas con los siguientes métodos:

Reducir la fuerza y relajar el agarre. Si el trabajo supone el uso de una caja registradora o de un teclado, por ejemplo, presiona las teclas suavemente. Para escribir a mano durante un tiempo 


\section{Síndrome de túnel carpiano}

Vol. 3, núm. 2., (2019)

Kruzcaya Monserrath Arévalo Sánchez; Ronald Roberto Reyes Sánchez; Marjorie Jacqueline Ramírez Ortiz; Carlos Xavier Villavicencio Bourne

prolongado, usar una lapicera grande que tenga un adaptador de agarre suave y de gran tamaño, así como tinta fluida.

Tomar descansos frecuentes. Estirar y doblar las manos y las muñecas suavemente de manera periódica. Cuando sea posible, cambiar de tarea. Es especialmente importante cuando se usan equipos que vibran o que requieren que ejerzas mucha fuerza.

Cuidar la posición. Evitar doblar demasiado la muñeca hacia arriba o hacia abajo. Una posición media y relajada es lo mejor. Mantener el teclado a la altura del codo o ligeramente por debajo de ese nivel.

Mejorar la postura. Con una postura incorrecta, los hombros se ubican hacia adelante, por lo que se acortan los músculos de los hombros y del cuello, se comprimen los nervios de este último. Esto puede afectar las muñecas, dedos y manos.

Cambiar el mouse de la computadora. Asegurarse de que el mouse de la computadora sea cómodo y no provoque tensión en la muñeca.

Mantener las manos calientes. Es más probable padecer dolor y rigidez en las manos cuando se trabaja en un lugar frío. Si no se puede controlar la temperatura en el lugar de trabajo, usar guantes sin dedos que mantienen las manos y las muñecas calientes.

Al continuar con la descripción de los diferentes aportes, se encuentra lo indicado por Garayora (2016), quien precisa que el síndrome del túnel carpiano consiste en una presión excesiva en el nervio mediano, que se extiende desde el antebrazo hasta la mano y es el que permite la 


\section{Síndrome de túnel carpiano}

Vol. 3, núm. 2., (2019)

Kruzcaya Monserrath Arévalo Sánchez; Ronald Roberto Reyes Sánchez; Marjorie Jacqueline Ramírez Ortiz; Carlos Xavier Villavicencio Bourne

sensibilidad y el movimiento a partes de la mano. Entre otros problemas, esta enfermedad puede provocar entumecimiento, hormigueo, debilidad o daño muscular en la mano y dedos. Debido a que este síndrome suele aparecer en profesionales de sectores que están muy feminizados, la presencia de esta enfermedad es nueve veces mayor en mujeres que en hombres. Además, complementa que el $88 \%$ de los trabajadores españoles tienen alguna dolencia.

Otro aporte de significación dada por este último autor se encuentra caracterizado por un $26 \%$ de los trabajadores tienen algún tipo de dolencia en muñecas y brazos, principalmente en los sectores del comercio o en ámbitos de trabajo donde se utilizan ordenadores; un $31 \%$ utiliza reposamuñecas para prevenir enfermedades y problemas como podría ser el síndrome del túnel carpiano. De igual manera, mostró que el $78 \%$ de las mujeres padecen este síndrome, debido a que el túnel carpiano en sí mismo puede ser más pequeño en la mujer que en el hombre. No se puede dejar de mencionar que durante esta etapa de la vida comienzan a aparecer los cambios de osteoartrosis en la mujer, favorecidos por la marcada disminución de los estrógenos; existencia de otros factores de tipo cultural; el elevado número de mujeres vinculadas al trabajo y la diversidad de labores, principalmente manuales, que realizan en la jornada laboral y cuando culminan esta; además de la intensa actividad doméstica

Cuadro $\mathbf{N}^{\circ} 1$ Relación Edad y Síndrome Túnel Carpiano

\begin{tabular}{|c|c|c|}
\hline Grupo de Edades & Pacientes & Frecuencia \% \\
\hline $21-30$ & 2 & 4 \\
\hline $31-40$ & 14 & 28 \\
\hline $41-50$ & 20 & 40 \\
\hline $51-60$ & 9 & 18 \\
\hline $61-70$ & 5 & 10 \\
\hline
\end{tabular}

Fuente: Elaboración Propia (2019) 


\section{Síndrome de túnel carpiano}

Vol. 3, núm. 2., (2019)

Kruzcaya Monserrath Arévalo Sánchez; Ronald Roberto Reyes Sánchez; Marjorie Jacqueline Ramírez Ortiz; Carlos Xavier Villavicencio Bourne

La distribución por edades se muestra un predominio en el grupo de 41 a 50 años de edad con 20 pacientes (40\%). La incidencia de síndrome de túnel carpiano STC aumentó con la edad hasta llegar a este grupo, donde el incremento se hace más notable. A esta edad se producen cambios degenerativos de articulaciones y tendones, unido a que todavía hacen actividades que realizaban en edades más tempranas y ello eleva el riesgo de padecer la enfermedad. Al sobrepasar los 50 años, aunque progresan los cambios degenerativos, el número de pacientes decrece, algo más marcado después de los 60 años, edades en las que aumenta el número de personas que se jubilan y disminuyen sus niveles de actividad. Además, a estas edades se diagnostica el mayor por ciento de enfermedades crónicas no transmisibles, que limitan la realización de determinadas labores y de su intensidad.

En esta misma dirección, se puede indicar que el síndrome del túnel del carpo (STC) es la neuropatía por atrapamiento más frecuente, con una incidencia y prevalencia que varían del $0,125 \%$ al $1 \%$ y del $5 \%$ al $16 \%$ respectivamente, en función de los criterios diagnósticos seleccionados. Se estima el riesgo de desarrollar un síndrome del túnel carpiano a lo largo de la vida en el 10\%, con una incidencia aproximada, en adultos, de 1 por cada 1000 personas. Numerosos estudios han demostrado un claro predominio en el sexo femenino, con un pico de incidencia entre los 55 a 60 años, con una proporción de 3- 1 frente a varones

\section{Conclusiones.}

Los aspectos que formaron parte del desarrollo de la revisión bibliográfica, para lograr construir un cuerpo de enfoques importantes relacionados con el síndrome de túnel carpiano, llevan a precisar una serie de aportes como mecanismos de reflexión, para así darle la respectiva 


\section{Síndrome de túnel carpiano}

Vol. 3, núm. 2., (2019)

Kruzcaya Monserrath Arévalo Sánchez; Ronald Roberto Reyes Sánchez; Marjorie Jacqueline Ramírez Ortiz; Carlos Xavier Villavicencio Bourne

consistencia, objetividad e innovación que el hecho investigativo requiere, entre ellas se consideran las siguientes:

La presencia del síndrome de túnel carpiano, representa hoy en día una enfermedad de tipo laboral, su sintomatología se encuentra determinada por la presencia de hormigueo, pérdida de la sensibilidad, disminución de la capacidad o fuerza que la mano, pueda tener para llevar a cabo una actividad cualquiera. Por ello, se busca como medida de prevención relajar los músculos del antebrazo y codo, de esta manera, los ligamentos pueden relajarse y así reducen la compresión interna que presentan el nervio carpiano. Por ser una enfermedad determinada por los movimientos repetitivos que realiza la mano dominante y la muñeca se puede indicar que la prevalencia del mismo es mayor en las mujeres cuyas edades oscilan entre 41-50 años respectivamente, pues, además del trabajo profesional u ocupacional, deben efectuar la labor doméstica. Se hacen presente cambios morfológicos en el nervio, los signos neurológicos son reversibles y los síntomas clínicos intermitentes: inicialmente sensitivos (dolor y parestesias) en el territorio del nervio mediano, fundamentalmente nocturnos por la tendencia de las muñecas a flexionarse durante el sueño y con los movimientos que impliquen flexoextensión del carpo y progresivamente tienden a hacerse continuos a pesar del reposo articular. Con relativa frecuencia el paciente puede referir síntomas sensitivos hasta el codo y más rara vez hasta el hombro. El síndrome suele iniciarse en el lado dominante, desarrollando posteriormente afectación bilateral. A nivel anatomopatológico, inicialmente, se observan alteraciones en el tejido conectivo como engrosamiento y desmielinización segmentaria fundamentalmente en las fibras de mayor tamaño más periféricas. A medida que avanza la compresión, la desmielinización es mayor, con degeneración axonal, con disminución de las fibras de mayor tamaño. En casos graves también pueden desarrollarse 


\section{Síndrome de túnel carpiano}

Vol. 3, núm. 2., (2019)

Kruzcaya Monserrath Arévalo Sánchez; Ronald Roberto Reyes Sánchez; Marjorie Jacqueline Ramírez Ortiz; Carlos Xavier Villavicencio Bourne

alteraciones proximales al segmento comprimido, con cambios retrógrados en el axón y pérdida de número de fibras gruesas.

Debido al incremento progresivo de la sintomatología y los signos físicos suelen acompañarse de forma paralela al deterioro en los parámetros electrodiagnósticos que permiten lograr la clasificación en leve, moderado o grave éste último es el que determina la utilización de procesos quirúrgicos. Inicialmente, existe disminución en la velocidad de conducción del segmento afecto, que puede progresar hasta existir un bloqueo completo. En estadios muy evolucionados el estudio de aguja puede mostrar potenciales de denervación en los músculos dependientes del nervio mediano e, incluso, disminución en la velocidad de conducción en el segmento proximal a la compresión.

\section{Bibliografía.}

Callejo, M. (2019). Investigación Científica. España: Ariel.

Garayova, S. (2016). Cirugía Ortopédica y Traumatología. Efe Salud, 36-43.

León, J. (2015). Fisioterapia de la Mano. Elportalsalud, 26-29.

Mora, L. (2019). Metodología de la Investigación Científica. México: Paidós.

Quiroz, P \& Hernández, R. (2017). Efectividad del Ultrasonido en el Síndrome de Túnel Carpiano. Cochrane. 11-18.

Silva, O. (2018). Síndrome del Túnel Carpiano. Médica Española, 6-12.

$$
\text { (c) (1) (8) (2) }
$$

\section{RECONOCIMIENTO-NOCOMERCIAL-COMPARTIRIGUAL}

CC BY-NC-SA

ESTA LICENCIA PERMITE A OTROS ENTREMEZCLAR, AJUSTAR Y CONSTRUIR A PARTIR DE SU OBRA CON FINES NO COMERCIALES, SIEMPRE Y CUANDO LE RECONOZCAN LA AUTORÍA Y SUS NUEVAS CREACIONES ESTÉN BAJO UNA LICENCIA CON LOS MISMOS TÉRMINOS. 